\title{
REGENERATIVE MEDICINE AND TISSUE ENGINEERING
}

\author{
RANIERI CANCEDDA *
}

\begin{abstract}
Tissue engineering and regenerative medicine constitute an emerging multidisciplinary field involving biology, medicine and engineering which is supposed to provide an outstanding contribution to improve the health and quality of life for million of people by restoring, maintaining or enhancing tissue and organ function. In regenerative medicine stem cells are playing a fundamental role: they are characterised by the ability to renew themselves through mitotic cell division and differentiating into a diverse range of specialized cell types. The two broad types of mammalian stem cells are embryonic stem cells that are found in blastocysts, and adult stem cells that are found in adult tissues. In the last few years, the use of stem cells has been proposed for tissue engineering. In conjunction with regenerative medicine it represents a new area of research aimed to restore tissues and organs which have been damaged by traumas, tumors, infective or degenerated pathologies. In the present paper, starting from the outstanding improvement of the basic knowledge recently obtained, the perspectives in this field are illustrated considering also the involved ethical issues and particular attention is paid to the financial and organizational problems which stay at the basis of the industrial development of such technologies.
\end{abstract}

\section{LA RICERCA}

La cellula staminale è definita come una cellula in grado di riprodursi per lunghi periodi e dare origine alle cellule specializzate che formano

* Università di Genova. 
i tessuti e gli organi. Cellule staminali sono state ottenute da embrioni, tessuti fetali, tessuti adulti. Negli ultimi anni l'utilizzo di cellule staminali è stato proposto nella medicina rigenerativa e per l'ingegneria dei tessuti. Queste due nuove branche della medicina rappresentano una area di ricerca che studia come riparare e rigenerare tessuti ed organi danneggiati da traumi, tumori, patologie da agenti infettivi o di tipo degenerativo, utilizzando i componenti ed i segnali naturali di un organismo (cellule staminali, fattori di crescita, ecc.). Sono state sollevate importanti questioni morali sull'utilizzo di cellule staminali umane. In alcuni casi le questioni poste sono più che legittime, ma in altri casi le perplessità derivano esclusivamente da una cattiva informazione. Vorrei pertanto fare una breve introduzione scientifica sull'argomento.

Anche se impropriamente, con il termine cellule staminali embrionali si intendono sia le cellule staminali embrionali vere e proprie sia le cellule germinali embrionali. Le cellule staminali embrionali derivano dalla massa cellulare interna della blastocisti, l'embrione precoce (dal $4^{\circ}$ al $5^{\circ}$ giorno). Molte delle conoscenze sulle cellule staminali embrionali sono state acquisite tramite ricerche su animali. In laboratorio queste cellule sono in grado di proliferare indefinitamente. Le cellule germinali embrionali derivano invece da tessuto fetale. Vengono isolate dalle cellule germinali primordiali dell'abbozzo delle gonadi del feto di $5-10$ settimane. In una fase più avanzata dello sviluppo fetale, l'abbozzo delle gonadi è destinato a trasformarsi nei testicoli o nelle ovaie e le cellule germinali primordiali daranno origine agli ovuli o allo sperma. Sia le cellule staminali che le cellule germinali embrionali sono pluripotenti, ossia danno origine a tipi di cellule differenziate derivate da tutti i tre i tessuti primitivi dell'embrione (endoderna, mesoderma ed ectoderma).

Le cellule staminali adulte sono invece cellule indifferenziate (non specializzate), presenti nei tessuti di organismi già sviluppati, ed in grado di dividersi in due cellule dando origine contemporaneamente a una replica della cellula indifferenziata originale ed ad una cellula specializzata con le caratteristiche delle cellule del tessuto di appartenenza. Per essere classificata come cellula staminale adulta, una cellula dovrebbe essere in grado di autoreplicarsi per tutto l'arco della vita dell'organismo. Questo criterio, per quanto fondamentale per la natura di una cellula staminale, è difficilmente dimostrabile in vivo. Nel corpo le cellule staminali adulte mantengono costanti le funzioni di un tessuto (omeostasi) garantendo il fisiologico ricambio cellulare ed in alcuni casi 
sostituendo anche le cellule che muoiono a causa di traumi o malattie. Sono rare, si disperdono nei tessuti dell'animale maturo e si comportano in modo molto diverso a seconda dell'ambiente che le circonda. I tessuti adulti nei quali è stata dimostrata la presenza di cellule staminali comprendono il midollo osseo, il sangue periferico, il cervello, il midollo spinale, la polpa dentale, i vasi sanguigni, i muscoli dello scheletro, gli epiteli dell'epidermide e dell'apparato digestivo, la cornea, la retina, il fegato ed il pancreas. Non è chiara l'origine delle cellule staminali adulte. È stato proposto che siano cellule che nel corso dello sviluppo abbiano mantenuto alcune delle caratteristiche delle cellule embrionali. Alcune cellule staminali adulte sembrano in grado di differenziarsi in tessuti diversi da quelli di origine. Per esempio le cellule stromali di midollo osseo si differenziano in cellule del muscolo cardiaco, dei muscoli scheletrici, del tessuto adiposo, dell'osso, cartilagine e del sistema nervoso. Questa capacità viene chiamata plasticità.

Grazie alla loro pluripotenzialità e plasticità, sia le cellule staminali embrionali che quelle adulte rappresentano una fonte ideale di cellule da usare per la riparazione di tessuti e organi danneggiati. Le cellule staminali embrionali, ma non le cellule staminali da adulto, quando vengono impiantate direttamente come cellule indifferenziate in topi con un sistema immunitario compromesso in modo da evitare la reazione di rigetto delle cellule impiantate, generano tumori benigni chiamati teratomi. A causa del rischio di crescita incontrollata, prima di poter prendere anche solo in considerazione l'impiego di cellule staminali embrionali per la ricostruzione di tessuti umani trapiantabili, è quindi indispensabile mettere a punto le tecnologie sia per indirizzare il differenziamento di queste cellule verso tipi cellulari specifici, sia per la loro separazione dalle cellule rimaste indifferenziate. L'utilizzo di cellule staminali embrionali pone rilevanti problemi etici. L'utilizzo di cellule staminali adulte è generalmente ben accetto dalla società.

Sulla base delle considerazioni di cui sopra, nei laboratori dell'IST abbiamo preferito rivolgere la nostra attività di ricerca all'identificazione, allo studio e all'utilizzo di cellule staminali e/o progenitrici adulte autologhe, ossia derivate dai tessuti dello stesso ricevente il trapianto.

Già a partire dalla metà degli anni 80 , nei nostri laboratori sono state messe a punto le condizioni per espandere in coltura cellule staminali da epiteli umani e ricostruire "in vitro" tessuti trapiantabili (epidermide, mucosa uretrale, epitelio corneale). Più recentemente l'attività si è rivolta prevalentemente alla rigenerazione ed alla riparazione dei 
tessuti scheletrici: cartilagine ed osso mediante l'utilizzo di cellule espanse "ex vivo" a partire da una piccola biopsia di cartilagine articolare e/o dal midollo osseo. Le cellule stromali da midollo osseo sono una popolazione cellulare con caratteristiche d'elevato interesse applicativo (terapia cellulare/genica). Particolare rilevanza deve essere attribuita alle condizioni di coltura per isolare ed espandere queste cellule che sono in grado di differenziare in diversi tipi cellulari dei tessuti connettivi tra cui osso, cartilagine, tendini, tessuto adiposo, muscolo.

Facendo tesoro dell'esperienza accumulata inizialmente una sperimentazione preclinica in animali di grossa taglia, è stata avviata una sperimentazione clinica per la ricostruzione ossea in pazienti con deficit di segmenti ossei a seguito di gravi traumi. I risultati di questa sperimentazione iniziata, prima al mondo, nel giugno 1998 sono stati pubblicati sul New England Journal of Medicine. In questo lavoro viene descritta la riparazione di gravi deficit, fino a $8 \mathrm{~cm}$, nelle ossa lunghe (tibia, ulna e omero) di tre pazienti mediante l'impiego di cellule staminali adulte prelevate dal midollo osseo dello stesso paziente ed espanse in vitro.

\section{TRASFERIMENTO INDUSTRIALE}

Per quanto riguarda il trasferimento di queste tecnologie all'industria, si dovrà facilitare la nascita di nuove società con un interesse per aspetti specifici della medicina rigenerativa come l'ingegneria dei tessuti, la terapia cellulare, lo sviluppo di nuovi biomateriali e le terapie innovative. A questo proposito deve essere tenuto presente che:

- le società di "start up" richiedono mediamente circa 1,5 - 3 milioni di euro per l'avvio delle attività (la partecipazione di partner industriali è importante);

- sono necessari grossi investimenti in strutture per garantire l'assenza di contaminazioni da agenti avventizi (batteri, funghi, virus);

- i prodotti richiedono circa 5-10 anni prima di raggiungere il mercato;

- gli obblighi imposti dalla regolamentazione attuale e da quella che sarà implementata a livello nazionale ed europeo non devono essere sottovalutati e possono condizionare significativamente i tempi necessari per raggiungere il mercato;

- gli aspetti bioetici devono essere presi in considerazione anche per la definizione della strategia di mercato (accettazione da parte dell'opinione pubblica). 
È allo studio la costituzione di una nuova società. Nei mese di agosto 2001 è stata inoltrata al MIUR domanda di finanziamento di un progetto di ricerca industriale a valere sul Fondo per le Agevolazioni per la Ricerca (FAR) che dovrà essere svolto dalla società (spin-off accademico) denominata "BIO-Rigen". È stato individuato nel laboratorio presso l'Istituto Nazionale per la Ricerca sul Cancro il referente scientifico in possesso della tecnologia da trasferire. I laboratori dell'IST hanno già ricevuto finanziamenti sia nazionali che europei per lo sviluppo di tecnologie trasferibili alle imprese nel settore di riferimento. La nuova impresa operante nelle biotecnologie applicate al settore ortopedico dovrebbe sviluppare da un punto di vista industriale il know how già esistente e quello che sarà raggiunto in futuro.

Hanno dimostrato interesse all'iniziativa due società straniere. Una di queste è attiva nel settore della produzione e certificazione dei terreni di coltura per uso umano. L'altra è produttrice di un biomateriale riassorbibile utilizzabile per applicazioni ortopediche. Le società stanno considerando una loro partecipazione sia finanziaria, sia mediante conferimento di know-how. Sono in corso, contatti con alcune società di "venture capital" per garantire il finanziamento dell'impresa.

\section{LA FORMAZIONE}

La nuova legge di riforma universitaria, recentemente pubblicata sulla Gazzetta Ufficiale prevede due livelli di laurea ed introduce per la prima volta in Italia le lauree in Biotecnologie.

La laurea di I livello ha una durata triennale con un titolo finale spendibile in tempi brevi nel mondo del lavoro. Nel corso dei primi due anni viene privilegiata la formazione di base, mentre nel terzo anno vengono privilegiate le attività biotecnologiche qualificanti e gli stage nelle industrie e nei centri di ricerca. A Genova il Corso di studi è stato attivato come Diploma Universitario nell'anno accademico 2000-01 e sarà trasformato in Corso di laurea a partire dall'anno accademico 2002-03. La laurea specialistica di II livello dovrà formare laureati in grado di gestire autonomamente attività di ricerca, sviluppo, produzione e controllo di qualità nel settore specifico. È in corso all'interno dell'Ateneo genovese un'approfondita discussione per la definizione dei contenuti dei corsi di laurea specialistici. In questa fase il contributo del mondo produttivo è non solo gradito, ma sollecitato. Si prevede di offrire un corso 
di laurea specialistico orientato verso la "Medicina Rigenerativa". Il corso dovrebbe essere attivato a partire dall'anno accademico 2003-04. Per la realizzazione dei progetti formativi previsti dai corsi di laurea in biotecnologie di I e II livello è necessario potenziare le attuali strutture didattiche per poter accogliere gli studenti nel modo migliore, soprattutto per quanto riguarda laboratori, biblioteche ed accesso a strumenti scientifici. In tutti i casi il numero massimo di studenti previsti per ciascun anno di corso della laurea di I livello è stato fissato in 30-40. Un numero ancora inferiore è previsto per le lauree specialistiche di II livello. Anche l'attuale corpo docente dovrà essere potenziato. Questa esigenza riguarda soprattutto l'attivazione delle lauree specialistiche di II livello per le quali sono necessarie competenze attualmente non esistenti nell'ateneo genovese.

Tutto questo comporta la necessità di nuovi investimenti da parte dell'amministrazione universitaria, e, ci si augura, da parte delle realtà produttive locali dove in un futuro dovranno inserirsi i futuri biotecnologi.

\section{CONCLUSIONI}

Le biotecnologie possono dare un contributo importante al futuro sviluppo di Genova a condizione che Genova divenga una delle capitali del mondo in settori specifici delle biotecnologie individuati sulla base delle eccellenze già esistenti nel territorio. Particolarmente interessante sembra essere il settore della Medicina Rigenerativa. Per rendersi conto di quanto una città si possa avvantaggiare da iniziative di questo tipo, vale la pena di approfondire quanto è stato fatto nella città di Pittsburg in USA che ha attraversato una crisi industriale simile a quella di Genova.

Se per il futuro sviluppo di Genova si vuole puntare anche sulle Biotecnologie, e sulla Medicina Rigenerativa in particolare, è necessario:

- Collaborare con l'Accademia per favorire sia la nascita di nuovi centri/istituti di ricerca pluridisciplinari, ma allo stesso tempo monotematici, sia l'arruolamento di nuovi ricercatori provenienti da altre regioni italiane e/o dall'estero.

- Favorire il trasferimento dai centri/punti di eccellenza all'industria mediante la messa a disposizione di risorse e facilitazioni per la creazione di nuove imprese (start up).

- Promuovere la formazione a tutti i livelli. In questo senso deve essere sottolineata la nascita del nuovo corso di laurea triennale in Biotecnologie e la prevista futura attivazione dei corsi di laurea specialistici. 


\section{BIBLIOGRAFIA}

Lemoli R.M., Bertolini F., Cancedda R., De Luca M., Del Santo A., Ferrari G., Ferrari S., Martino G., Mavilio F., Tura S., "Stem cell plasticity: time for a reappraisal?”, Haematologica, 90(3): 360-81, March 2005.

Cancedda R., Bianchi G., Derubeis A., Quarto R., "Cell therapy for bone disease: a review of current status”, Stem Cells, 21(5): 610-9, 2003.

Cancedda R., Dozin B., Giannoni P., Quarto R., "Tissue engineering and cell therapy of cartilage and bone”, Matrix Biol, 22(1): 81-91, March 2003.

Quarto R., Mastrogiacomo M., Cancedda R., Kutepov S.M., Mukhachev V., Lavroukov A., Kon E., Marcacci M., "Repair of large bone defects with the use of autologous bone marrow stromal cells", N Engl J Med, 344(5): 385-6, February 2001.

Mathor M.B., Ferrari G., Dellambra E., Cilli M., Mavilio F., Cancedda R., De Luca M., "Clonal analysis of stably transduced human epidermal stem cells in culture”, Proc Natl Acad Sci U S A, 93(19): 10371-6, September 1996. 
